\title{
Refuge
}

Canada's Journal on Refugees

Revue canadienne sur les réfugiés

\section{The Criminalization of Migration: Context and Consequences dirigé par Idil Atak et James C. Simeon}

\section{David Moffette and Martha Vargas Aguirre}

Volume 35, Number 2, 2019

Private Sponsorship in Canada

URI: https://id.erudit.org/iderudit/1064826ar

DOI: https://doi.org/10.7202/1064826ar

See table of contents

Publisher(s)

Centre for Refugee Studies, York University

ISSN

0229-5113 (print)

1920-7336 (digital)

Explore this journal

Cite this review

Moffette, D. \& Vargas Aguirre, M. (2019). Review of [The Criminalization of Migration: Context and Consequences dirigé par Idil Atak et James C. Simeon] Refuge, 35(2), 126-127. https://doi.org/10.7202/1064826ar 


\title{
The Criminalization of Migration: Context and Consequences \\ $\sim$
}

\author{
Dirigé par Idil Atak et James C. Simeon \\ Montréal et Kingston: McGill-Queen’s University Press, 2018, 423 pp.
}

$\mathrm{L}$ e discours populiste et anti-immigrant du président américain et la crise politique de l'Union Européenne présentée comme une crise des réfugiés - ont généralisé pour le grand public l'idée que nous vivons une période de sécurisation et de criminalisation de l'immigration et du refuge. À la veille des élections fédérales canadiennes de 2019, le gouvernement libéral - qui avait pourtant opéré un changement de cap important en matière de discours sur la migration - semble maintenant vouloir satisfaire un électorat conservateur en resserrant l'Accord sur les tiers pays sûrs, une forme claire de "néo refoulement» (Hyndman et Mountz 2008). Après une décennie active de politiques répressives du précédent gouvernement conservateur, il semble donc que la tendance coercitive de fond continue aussi au Canada.

Il ne fait ainsi aucun doute que le moment actuel est marqué par une criminalisation de l'immigration. D’ailleurs, comme le soulignent James C. Simeon et Idil Atak en conclusion de l'ouvrage, on note dans plusieurs pays un recours de plus en plus fréquent au droit criminel pour punir des stratégies migratoires, ainsi que l'imposition de sanctions migratoires comme conséquences d'un dossier criminel. Pourtant, malgré ce que le populaire néologisme «crimmigration» peut laisser supposer, les intersections entre le droit pénal et le droit de l'immigration sont multiformes, et la notion de criminalisation est elle-même polysémique. Comme l'indiquait déjà Weber en 2002, le terme peut faire référence à une criminalisation formelle (c'est à dire l'interdiction de certaines pratiques migratoires par le droit pénal), une quasi-criminalisation ou criminalisation procédurale (comme dans le cas de la détention administrative), et une criminalisation rhétorique (qui est souvent, mais pas toujours, articulée conjointement aux deux autres formes). Puis, si la situation politique globale actuelle laisse plusieurs analystes pessimistes, la criminalisation actuelle n'est pas nouvelle, mais s'inscrit dans le long tournant restrictionniste des 20 à 30 dernières années.

L'heure est donc aux bilans et ce livre offre, en ce sens, une contribution édifiante. Au premier regard, plusieurs éléments attirent l'attention sur cet ouvrage collectif. Le titre, d'abord, qui promet un survol exhaustif de la question, mais surtout la liste impressionnante des auteurs dont l'expérience et l'expertise ne font aucun doute. Par exemple, le fait que collectivement les auteurs aient contribué - à titre d'avocats ou de conseillers juridiques - à plusieurs des cas emblématiques récents entendus à la Cour suprême du Canada et qui ont marqué la jurisprudence sur des questions liées à la criminalisation de l'immigration et du refuge: Appulonappa et Bo1o (Grant), Tran (Shazadi Meighen) et Ezokola (Waldman). Joseph Rikhof - un pionnier des travaux sur ce sujet - y a aussi un chapitre, et François Crépeau - l'ancien Rapporteur des Nations Unies sur les droits de l'homme des migrants (2011-2017) - signe la préface. D’autres auteurs ont aussi été actifs à la Commission de l'immigration et du statut de réfugié du Canada ou son équivalent britannique (Gleeson, Simeon, Weisman).

Idil Atak et James C. Simeon - qui ont en commun d'avoir siégé sur l'exécutif de l'Association canadienne d'études sur les réfugiés et la migration forcée, dont un colloque a mené au projet de livre - ont ainsi rassemblé les contributions de 16 chercheurs reconnus. Si la plupart des auteurs sont juristes et plusieurs des chapitres offrent des commentaires sur le droit, on retrouve aussi des texte plus philosophiques, criminologiques, sociologiques et historiques que nous avons beaucoup appréciés (Ben-Arieh, Horner, Godspeed, Hannan et Bauder, Hudson, Molnar). En particulier, les chapitres basés sur des entretiens ou sur un travail en archives ajoutent une richesse empirique qui rend la lecture très agréable tout en permettant doffrir une grille de lecture novatrice et de dégager des tendances que l'analyse des politiques officielles tend à perdre de vue.

Louvrage collectif rassemble donc divers chapitres sous le thème très général de criminalisation de l'immigration, offrant ainsi un genre de «reader» qui est le bienvenu. Les étudiants en droit de l'immigration et leurs enseignants, les personnes qui veulent mieux connaitre les formes que prend la criminalisation de l'immigration et du refuge au Canada (objet de la majorité des chapitres), ainsi que les chercheurs qui travaillent sur l'exclusion de la protection en vertu de l'alinéa $1 \mathrm{~F}$ de la Convention de 1951 et de l'arrêt Ezokola (traitée par le tiers des chapitres) trouveront dans ce livre un survol exhaustif des enjeux importants ainsi que des clés de lecture pour saisir les implications juridiques et politiques de ces développements.

En effet, les textes apportent tous une perspective originale et sont de bonne facture. Il n'y a pas, à notre avis, de chapitre 
dont la qualité contrasterait avec l'ensemble. La répartition inégale des approches, enjeux, et situations géographiques donne cependant l'impression que les chapitres sont inégalement intégrés, et les thèmes qui englobent chacune des sections semblent parfois un peu aléatoires. En effet, mise à part la Section 2 sur Ezokola et l'exclusion des réfugiés qui est très bien intégrée thématiquement, il est difficile d'identifier le fil conducteur qui unit les contributions dans chacune des sections. De même, comme la plupart des chapitres portent sur des cas canadiens, les textes qui divergent de cette tendance pour s'intéresser à la Turquie, à l'Union Européenne, ou aux États-Unis - tous très intéressant en eux-mêmes - se retrouvent tout de même un peu isolés. Par ailleurs, si Graham fait un travail franchement remarquable de problématisation des notions de "crimmigration" et de criminalisation et questionne la pertinence de l'analogie comme stratégie pour contrer les violences causées par le contrôle de l'immigration, et si Simeon et Atak offrent en conclusion une discussion de ces notions, plusieurs des auteurs ne définissent pas vraiment ce qu'ils entendent par criminalisation, crimmigration ou sécurisation, et les concepts semblent parfois interchangeables et vagues.

Cela dit, c'est le défi que rencontrent tous les ouvrages collectifs: il faut choisir entre une collection très intégrée mais un peu pointue, et un ouvrage de plus grande amplitude mais plus diversifié. Le choix des directeurs de ce livre d’opter pour la deuxième option - malgré les limites inhérentes au genre - a clairement porté fruit. Ce premier titre de la nouvelle série McGill-Queen's Refugee and Forced Migration Studies est un choix parfait pour lancer cette collection et devrait nous inciter à suivre avec intérêt le futur de la série. Un livre pertinent qui tombe à point et représente une contribution importante aux débats contemporains.

\section{RÉFÉRENCES CITÉES}

Hyndman, Jennifer, et Alison Mountz. 2008. «Another Brick in the Wall? Neo-Refoulement and the Externalization of Asylum by Australia and Europe». Government and Opposition 43(2): 249-69.

Weber, Leanne. 2002. "The Detention of Asylum Seekers: 20 Reasons Why Criminologists Should Care». Current Issues in Criminal Justice 14(1): 9-30.

David Moffette est professeur adjoint au Département de criminologie de l'Université d'Ottawa. Martha Vargas Aguirre est doctorante au Département de criminologie de l'Université d'Ottawa. On peut joindre les auteurs à david.moffette@ uottawa.ca.

\title{
Refugee Resettlement: Power, Politics, and Humanitarian Governance \\ $\sim$
}

\author{
Edited by Adèle Garnier, Liliana Lyra Jubilut, and Kristin Bergtora Sandvik \\ Oxford, UK: Berghahn, 2018, 317 pp.
}

$\mathrm{I}$ $\mathrm{n}$ the first decades after the Second World War, refugee policy meant resettlement and foreign aid. Since the 1980 , resettlement took a back seat to repatriation and asylum as dominant themes within refugee policy discourse. And now, it seems that it is back.

Since 2015, when the global refugee crisis commanded a greater share of international news, expanding resettlement has been seen as an important part of the solution. The Global Compact on Refugees, affirmed in December 2018 by the UN General Assembly, identified expanded access to third-country solutions as a key objective of international cooperation.

For this reason, Refugee Resettlement: Power, Politics, and Humanitarian Governance is timely. Few volumes have studied refugee resettlement within an international comparative framework. This alone makes the book worthwhile: to obtain a perspective on third-country solutions with a wider set of cases and longer time horizon than other books on resettlement in the United States, Canada, and Australia - the three primary destinations for resettled refugees.

The volume's organizing concept is "humanitarian governance," the ways in which refugee resettlement involves both care for the vulnerable and control over their lives. In the introductory chapter, the editors describe how this concept directs the analysis of the chapters that follow, as they consider the ways in which power operates in resettlement. Resettlement works in a multi-level system in which international organizations, national governments, and other agencies all shape the journeys and opportunities of refugees moving through resettlement. 